\title{
Telomere Capping
}

National Cancer Institute

\section{Source}

National Cancer Institute. Telomere Capping. NCI Thesaurus. Code C20339.

Processes that prevent chomosome ends from degradation and from being recognized as a double strand break. 